\title{
The stromelysin-1 5A/6A promoter polymorphism is a disease marker for the extent of coronary heart disease
}

\author{
Adelheid Schwarz ${ }^{\mathrm{a}}$, Werner Haberbosch ${ }^{\mathrm{b}}$, Harald Tillmanns ${ }^{\mathrm{b}}$ and Andreas Gardemann ${ }^{\mathrm{a}, *}$ \\ ${ }^{\mathrm{a}}$ Institut für Klinische Chemie und Pathobiochemie and \\ ${ }^{\mathrm{b}}$ Abteilung Kardiologie und Angiologie, Justus-Liebig-Universit ät Giessen, Germany
}

\begin{abstract}
Background. Matrix metalloproteinases, such as stromelysin-1, are implicated in the pathogenesis of coronary artery disease (CAD) and acute myocardial infarction (MI). A 5A/6A promoter polymorphism can regulate the transcription of the stromelysin-1 gene in an allele-specific manner. Evidence has been presented that the $6 \mathrm{~A}$ allele is associated with the progression of coronary heart disease (CHD). In contrast, the 5A allele may be linked to the risk of MI.

Results. To analyse the relation of the 5A/6A polymorphism with the risk and severity of CHD and the risk of MI, a case-control study of 515 healthy controls and 1848 participants who underwent coronary angiography for diagnostic purposes was conducted. In the total sample, the mean CHD scores - according to Gensini - were different between 5A/6A genotypes: 5A5A homozygotes had the lowest, 6A6A genotypes the highest and 5A6A heterozygotes intermediate scores. These differences were even more pronounced when the participants were restricted to individuals with a high coronary risk profile (high apoB levels, high $\mathrm{Lp}$ (a) levels, high glucose levels, combinations of either high apoB and Lp(a) levels or high apoB, Lp(a) and glucose plasma levels). Mean values were used as cut points for high-risk populations, respectively. In contrast, the $5 \mathrm{~A}$ allele was not associated with the risk of CHD or MI. Even when angiographically controlled individuals without MI were compared with MI patients in subpopulations of participants with no, single, double and triple vessel disease, the frequencies of the 5A/6A and/or the 5A5A genotypes were not higher in each subgroup, respectively.

Conclusions. The present results do not confirm an association of the 5A allele with the risk of MI, observed in another investigation, but strengthen the hypothesis of earlier studies that the $6 \mathrm{~A}$ allele is a disease marker for progression of coronary heart disease. Further investigations should evaluate whether 6A allele carriers and especially 6A homozygotes might benefit from a more aggressive therapy against CHD progression.
\end{abstract}

\section{Introduction}

Atherosclerosis is directly or indirectly related to nearly one half of all deaths in the Western world [1-3]. Life-threatening complications of coronary atherosclerosis are the acute coronary syndromes including unstable angina pectoris and acute myocardial infarction (MI) [4]. It is well known that plaque rupture with

*Address for correspondence: Pathologische Biochemie (Haus 20), Leipziger Strasse 44, D-39120 Magdeburg, Germany. Tel.: +49 391 6713640; Fax: +49391 6713639; E-mail: andreas.gardemann@ medizin.uni-magdeburg.de. subsequent thrombosis is a critical event in the pathogenesis of acute MI [4,5].

Matrix metalloproteinases (MMPs), such as stromelysin-1 (MMP-3), can degrade extracellular matrix and are localised extensively in coronary atherosclerotic plaques [6,7]. MMPs have been shown to be implicated in accelerating growth of coronary atheromas by remodelling connective tissue during atherogenesis [811]. In addition, MMPs may contribute to weakening of the plaque cap which provokes plaque rupture, thrombosis and consequently acute MI [6].

A bi-allelic single nucleotide polymorphism in the promoter region of the stromelysin-1 gene has been identified by Ye et al. [12]. The 5A allele is charac- 
terised by a cluster of 5 adenosines, the other by six adenosines (6A). The $5 \mathrm{~A}$ allelic stromelysin- 1 promoter has been shown to have greater transcription activity than the 6A allelic promoter [13]. Apparently, this difference is due to preferential binding of a transcription repressor to the $6 \mathrm{~A}$ allele $[14,15]$. The impact of this functional polymorphism on coronary heart disease (CHD) has been investigated only in few studies [13, 16-18]. The 6A allele has been found to be associated with accelerated growth of coronary atheromas [13, $16,17]$ and predisposition to post-angioplasty restenosis [17]. On the other hand, in a Japanese study sample, the $5 \mathrm{~A}$ allele was associated with an increased risk of MI [18]. However, this observation has not been checked by other investigators. In addition, studies investigating the potential association of the stromelysin1 promoter $5 \mathrm{~A} / 6 \mathrm{~A}$ polymorphism with the risk of coronary artery disease (CAD) have not been published so far. Therefore, we decided to analyse the impact of this gene polymorphism on the risk of CAD and MI and on the severity of coronary heart disease in a population of 515 healthy controls and 1848 patients who underwent coronary angiography for diagnostic purposes.

\section{Materials and methods}

\subsection{Detection of coronary artery disease and myocardial infarction}

\subsubsection{Coronary artery disease}

Coronary angiography was performed by the Judkins method; coronary vessels with at least $50 \%$ stenosis were defined as diseased. By means of coronary angiography, the study population was divided into subjects without any angiographic CAD or with coronary arterial stenoses less than 50\% (no vessel disease) and individuals with single, double or triple vessel disease. The severity of CHD was also estimated by calculating the Gensini score [19]; this score is designated "CHD score" in subsequent text.

\subsubsection{Myocardial infarction}

Acute MI was diagnosed according to criteria established by the World Health Organisation. Within the subgroup of angiographic controlled individuals, $16 \%$ of patients without vessel disease, $47 \%$ of individuals with single vessel disease, $51 \%$ of patients with double vessel disease and $62 \%$ of patients with triple vessel disease had suffered a MI before recruitment in the study.

\subsection{Study sample}

The gene polymorphism was analysed in 2363 male Germans. All individuals who agreed to participate in the study were evaluated with a structured questionnaire which provided information about coronary risk factors [20]. The study was approved by the local ethics committee.

For the control group of 515 male individuals without vascular disease, we used the detailed Rose questionnaire [20] to identify disease free controls and to exclude subjects who were suspicious to have any form of vascular disease. Controls had to be (a) without a history of MI or angina, (b) without symptoms, suggesting angina assessed by the Rose questionnaire [20], and (c) without a history of stroke or any other vascular event. Systematic screening of the control participants' families was conducted and subjects with a family history of premature coronary heart disease (presence of symptomatic ischemic heart disease or coronary death in a first-degree relative aged $<55$ years) were excluded from the study.

A group of 1848 participants underwent coronary angiography for diagnostic purposes. About $80 \%$ of the participants underwent this diagnostic procedure due to presumptive or verified CHD. The remaining part consisted almost completely of patients who underwent coronary angiography for clarification of reduced left ventricular function. In $90 \%$ of these patients, CAD was documented; in $10 \%$ of this subpopulation $(=2 \%$ of the total cohort), reduced left ventricular function was caused by dilated cardiomyopathy or longstanding arterial hypertension.

In the present study, established risk factors and the stromelysin 5A/6A genotypes were compared between (i) healthy controls without vascular disease and CAD patients (verified by coronary angiography), (ii) participants without and with CAD within the subpopulation of individuals with coronary angiography, (iii) subjects with different degrees of CAD (verified by coronary angiography), (iv) healthy controls without vascular disease and MI patients and (v) MI negative and MI positive patients within the subgroup of individuals with coronary angiography.

\subsection{Measurements of substrates, definition of variables and genotyping of stromelysin 5A/6A promoter}

Triglyceride, total cholesterol, apolipoprotein B (apoB), apolipoprotein AI (apoAI), lipoprotein (a) 
(Lp(a)), glucose, fibrinogen and body mass index (BMI) were measured or calculated as described [21]. Diabetes mellitus and hypertension are given as binary variables and not divided into subcategories. Hypertension was defined by either treatment or diastolic blood pressure higher than $95 \mathrm{~mm} \mathrm{Hg}$ on two consecutive visits for those untreated. Cigarette consumption was given as pack-years (1 pack-year: e.g. 20 cigarettes per day for one year).

The stromelysin 5A/6A promoter polymorphism was analysed according to the method of Dunleavey et al. [22]. In principle, three mismatched nucleotides were introduced in a PCR primer annealed to the proximity of the 5A/6A polymorphism, creating a recognition sequence for restriction endonuclease Xmn1 at the polymorphic site. An Xmn 1 digestion cleaved PCR products deriving from $5 \mathrm{~A}$ allele but not from $6 \mathrm{~A}$ allele [22].

\subsection{Definition of low and high risk subpopulations}

The mean values of coronary risk factors (age, apoAI, apoB, apoAI/apoB ratio, $\mathrm{Lp}(\mathrm{a})$, fibrinogen, cigarette smoking) from the study population were used as cut points for definition of low and high risk populations. With respect to hypertension and diabetes, low and high risk groups were defined by the absence or presence of these diseases. All low- and high-risk groups, presented in this study, were considered in our primary hypotheses.

\subsection{Statistical analysis}

Statistical analyses were performed using the SPSS for Windows software (Version 9.0). The $\chi^{2}$-test was used to test for deviation of genotype distribution from Hardy-Weinberg equilibrium. In order to compare established risk factors between stromelysin 5A/6A genotypes, the relation of this polymorphism to continuous variables was tested by Kruskal-Wallis 1-Way Anova and to diabetes and hypertension by $\chi^{2}$ analysis. Established risk factors for CAD and MI were identified by multiple regression analysis (extent of CAD, CHD score) or multiple logistic regression (absence /presence of CAD or MI). The relation of the $5 \mathrm{~A} / 6 \mathrm{~A}$ polymorphism to the extent of CAD was determined by multiple regression analysis with adjustment for coronary risk factors. Odds ratios with $95 \%$ confidence intervals as an estimate of relative risk of CAD and MI and two-tailed p-values were calculated by multiple logistic regression after adjustment for coronary risk factors. A two-sided probability value of less than 0.05 was considered to indicate statistical significance. The analyses were based on a recessive, co-dominant and dominant model of inheritance.

\section{Results}

\subsection{Distribution of the stromelysin 5A/6A genotypes and its effect on physiochemical and clinical variables}

A deviation of the stromelysin $5 \mathrm{~A} / 6 \mathrm{~A}$ genotypes from Hardy-Weinberg equilibrium was not observed in the total sample ( $p=0.249)$, in healthy controls without vascular disease ( $p=0.753)$, in angiographic controlled patients without MI $(p=0.908)$, without CAD $(p=0.941)$, without CAD and also MI $(p=0.902)$, and in patients with CAD ( $p=0.330)$ or with MI ( $p=$ 0.253). Age, total cholesterol, triglycerides, apoAI, apoB, apoAI/apoB ratio, Lp(a), glucose, fibrinogen, prevalence of diabetes and arterial hypertension, BMI and cigarette consumption were almost identical between $5 \mathrm{~A} / 6 \mathrm{~A}$ genotypes (i) of the total study population, (ii) of healthy controls, (iii) of patients without or with single- or multi-vessel disease and (iv) of subjects without and with MI (data not shown). The mean delay between the occurrence of MI and recruitment in this study ( $5.6 \pm 0.24$ years) did not differ between $5 \mathrm{~A} / 6 \mathrm{~A}$ genotypes ( $p=0.636$; data not shown).

\subsection{Relation of established risk factors to the risk of coronary artery disease and myocardial infarction and the extent of coronary heart disease}

\subsubsection{Risk of coronary artery disease}

A comparison of the control group of healthy individuals without vascular disease with angiographic proven CAD patients revealed apoB $(p=0.00001), \mathrm{Lp}(\mathrm{a})$ $(p=0.00001)$, diabetes mellitus $(p=0.0002)$, hypertension $(p=0.00001)$, smoking habit (pack-years, $p=0.0009)$ and age $(p=0.00001)$ as risk factors for $\mathrm{CAD}$, and apoAI ( $p=0.00001)$ and consequently high apoAI/apoB ratios $(p=0.00001)$ as protective factors against this disease (Table 1). Within the group of angiographic controlled patients, the same risk factors were identified (Table 1). 
Table 1

Distribution of risk factors of coronary artery disease and myocardial infarction.

\begin{tabular}{|c|c|c|c|c|c|c|c|c|c|c|}
\hline \multirow[t]{2}{*}{ Risk factor } & \multicolumn{5}{|c|}{ \pm Coronary artery disease } & \multicolumn{5}{|c|}{ \pm Myocardial infarction } \\
\hline & $\begin{array}{l}-\mathrm{CAD}^{(1)} \\
(n=515)\end{array}$ & $\begin{array}{l}-\mathrm{CAD}^{(2)} \\
(n=432)\end{array}$ & $\begin{array}{c}+ \text { CAD } \\
(n=1416)\end{array}$ & $2 \mathrm{p}^{(1)}$ & $2 \mathrm{p}^{(2)}$ & $\begin{array}{l}-\mathrm{MI}^{(1)} \\
(n=515)\end{array}$ & $\begin{array}{c}-\mathrm{MI}^{(2)} \\
(n=1003)\end{array}$ & $\begin{array}{c}+\mathrm{MI} \\
(n=845)\end{array}$ & $2 \mathrm{p}^{(1)}$ & $2 \mathrm{p}^{(2)}$ \\
\hline Age (years) & $55.3 \pm 10.3$ & $57.7 \pm 11.4$ & $62.8 \pm 9.4$ & 0.00001 & 0.00001 & $55.3 \pm 10.3$ & $61.0 \pm 10.5$ & $62.4 \pm 9.7$ & 0.00001 & 0.0004 \\
\hline BMI $\left(\mathrm{kg} / \mathrm{m}^{2}\right)$ & $26.3 \pm 3.3$ & $27.2 \pm 3.9$ & $27.0 \pm 3.4$ & n. s. & n. s. & $26.3 \pm 3.3$ & $27.1 \pm 3.6$ & $27.0 \pm 3.5$ & n. s. & n. s. \\
\hline Pack-years & $16.2 \pm 28$ & $20.5 \pm 25$ & $23.9 \pm 27$ & 0.0009 & 0.018 & $16.2 \pm 28$ & $20.5 \pm 25$ & $26.0 \pm 27$ & 0.00001 & 0.00001 \\
\hline$\%$ Diabetes & 8 & 13 & 21 & 0.0002 & 0.0055 & 8 & 18 & 21 & 0.0002 & n. s. \\
\hline$\%$ Hypertension & 16 & 57 & 68 & 0.00001 & 0.002 & 16 & 66 & 65 & 0.00001 & n. s. \\
\hline Chol (mg/dl) & $211 \pm 43$ & $202 \pm 42$ & $207 \pm 43$ & n. s. & n. s. & $211 \pm 43$ & $208 \pm 43$ & $204 \pm 44$ & n. s. & n. s. \\
\hline Trig (mg/dl) & $139 \pm 53$ & $137 \pm 83$ & $147 \pm 83$ & n. s. & n. s. & $139 \pm 53$ & $145 \pm 87$ & $145 \pm 78$ & n. s. & n. s. \\
\hline ApoAI (g/l) & $1.46 \pm 0.25$ & $1.42 \pm 0.27$ & $1.38 \pm 0.29$ & 0.00001 & 0.0003 & $1.46 \pm 0.25$ & $1.41 \pm 0.27$ & $1.37 \pm 0.30$ & 0.00001 & 0.0015 \\
\hline ApoB (g/l) & $1.11 \pm 0.26$ & $1.16 \pm 0.27$ & $1.25 \pm 0.32$ & 0.00001 & 0.00001 & $1.11 \pm 0.26$ & $1.22 \pm 0.30$ & $1.24 \pm 0.32$ & 0.00001 & n.s. \\
\hline ApoAI/ApoB & $1.39 \pm 0.43$ & $1.29 \pm 0.47$ & $1.23 \pm 0.31$ & 0.00001 & 0.00001 & $1.39 \pm 0.43$ & $1.22 \pm 0.41$ & $1.17 \pm 0.36$ & 0.00001 & 0.009 \\
\hline Lp(a) (mg/l) & $20 \pm 25$ & $21 \pm 28$ & $30 \pm 38$ & 0.00001 & 0.00001 & $20 \pm 25$ & $26 \pm 36$ & $29 \pm 36$ & 0.00001 & n. s. \\
\hline
\end{tabular}

Values are means \pm SD or $\%$ of a group. The relation of the risk factors to CAD and to MI was analysed by multiple logistic regression. Abbreviations: n. s., not significant; Chol, cholesterol; Trig, triglycerides; Apo, apolipoprotein; "-CAD" ${ }^{1)}$ " and "-MI(1)", control group of individuals free of cardiovascular disease; “-CAD ${ }^{(2)}$ ", group of patients with coronary angiography and without vessel disease; "-MI ${ }^{2)}$ ", patients with coronary angiography and without MI"; " $2 \mathrm{p}^{(1)}$ "; two-tailed p-values for the comparisons of CAD (MI) patients with control individuals $\left(“-\mathrm{CAD}^{(1)},-\mathrm{MI}^{(1)}\right.$ "); " $2 \mathrm{p}^{(2)}$ ", two-tailed p-values for the comparisons between patients who underwent coronary angiography.

\subsubsection{Extent of coronary artery disease, $C H D$ score}

With respect to the extent of CAD and to the CHD score, $\operatorname{apoB}(p=0.00001), \operatorname{Lp}(\mathrm{a})(p=0.00001)$, diabetes mellitus $(p=0.00001)$, hypertension $(p=$ $0.00001)$, smoking habit (pack-years, $p=0.018$ ) and age $(p=0.00001)$ were identified as risk factors and apoAI $(p=0.00001)$ and high apoAI/apoB ratios $(p=$ $0.00001)$ as protective factors (data not shown).

\subsubsection{Risk of myocardial infarction}

When the control group of healthy individuals without coronary heart disease was compared with MI patients, apoB $(p=0.00001), \operatorname{Lp}(\mathrm{a})(p=0.00001)$, diabetes mellitus $(p=0.0002)$, hypertension $(p=$ $0.00001)$, smoking habit $(p=0.00001)$ and age $(p=$ $0.00001)$ could be demonstrated as risk factors, and apoAI ( $p=0.00001)$ and high apoAI/apoB ratios $(p=$ $0.00001)$ as protective factors against MI (Table 1). When the study sample was restricted to angiographic controlled individuals, only smoking habit $(p=$ $0.00001)$ and age $(p=0.0004)$ were found as risk factors and apoAI $(p=0.0015)$ and high apoAI/apoB ratios $(p=0.009)$ as protective factors (Table 1$)$.

\subsection{Relation of the stromelysin 5A/6A promoter polymorphism to coronary heart disease}

\subsubsection{Risk of coronary artery disease}

With all models of inheritance, not any association was detected between the stromelysin $5 \mathrm{~A} / 6 \mathrm{~A}$ promoter polymorphism and the risk of CAD. This observation applied not only to the total sample (Table 2), but also to low- and high-risk populations (data not shown).

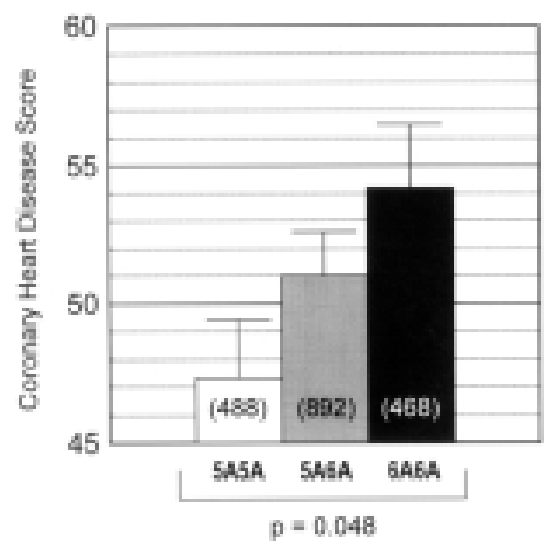

Fig. 1. Comparison of mean coronary heart disease scores between the stromelysin-1 promoter $5 \mathrm{~A} / 6 \mathrm{~A}$ genotypes in the total study sample. The mean CHD scores were compared by multiple regression analysis after adjustment for coronary risk factors between patients who underwent coronary angiography for diagnostic purposes. Values are means \pm SEM. Numbers of individuals are given in parentheses. Coronary angiography was performed by the Judkins method. The CHD score was estimated according to Gensini [19]. A two-tailed probability value of $<0.05$ was considered to indicate statistical significance.

\subsubsection{Extent of coronary artery disease, $C H D$ score}

In the total population, the $5 \mathrm{~A} / 6 \mathrm{~A}$ promoter polymorphism was not associated with the extent of CAD defined by the number of diseased vessels (Table 2). However, in the total sample the mean CHD scores were significantly different between $5 \mathrm{~A} / 6 \mathrm{~A}$ genotypes: $5 \mathrm{~A} 5 \mathrm{~A}$ homozygotes had the lowest, 6A6A genotypes the highest and 5A6A heterozygotes intermediate Gensini scores (Fig. 1; $p=0.048$ ). These differences were even more pronounced when the study participants were re- 
Table 2

Distribution of the stromelysin 5A/6A genotypes in subjects without or with coronary artery disease and individuals without or with acute myocardial infarction

\begin{tabular}{|c|c|c|c|c|c|c|c|}
\hline \multirow[t]{2}{*}{ Controls/Cases } & \multirow[t]{2}{*}{ Mean age $( \pm \mathrm{SD})$} & \multirow[t]{2}{*}{$\mathrm{n}$} & \multicolumn{3}{|c|}{ 5A/6A Genotypes } & \multicolumn{2}{|c|}{ 5A/6A Alleles } \\
\hline & & & $\mathrm{n}(5 \mathrm{~A} 5 \mathrm{~A})$ & $\mathrm{n}(5 \mathrm{~A} 6 \mathrm{~A})$ & $\mathrm{n}(6 \mathrm{~A} 6 \mathrm{~A})$ & $5 \mathrm{~A}(95 \% \mathrm{CI})$ & $6 \mathrm{~A}(95 \% \mathrm{CI})$ \\
\hline \multicolumn{8}{|l|}{$\pm \mathrm{CAD}$} \\
\hline \multicolumn{8}{|c|}{ Subjects without coronary angiography } \\
\hline No CAD (healthy controls) & $55.3 \pm 10.2$ & 515 & 139 & 249 & 127 & $0.51(0.48-0.54)$ & $0.49(0.46-0.52)$ \\
\hline \multicolumn{8}{|c|}{ Subjects with coronary angiography } \\
\hline No vessel disease & $57.7 \pm 11.4$ & 432 & 122 & 212 & 98 & $0.53(0.49-0.56)$ & $0.47(0.44-0.51)$ \\
\hline Single vessel disease & $60.7 \pm 9.8$ & 359 & 101 & 162 & 96 & $0.51(0.47-0.55)$ & $0.49(0.46-0.53)$ \\
\hline Double vessel disease & $62.9 \pm 9.8$ & 396 & 101 & 189 & 106 & $0.49(0.46-0.53)$ & $0.51(0.47-0.54)$ \\
\hline Triple vessel disease & $64.0 \pm 8.9$ & 661 & 164 & 329 & 168 & $0.50(0.47-0.52)$ & $0.50(0.48-0.53)$ \\
\hline \multicolumn{8}{|l|}{$\pm \mathrm{MI}$} \\
\hline \multicolumn{8}{|c|}{ Subjects without coronary angiography } \\
\hline No MI (healthy controls) & $55.3 \pm 10.2$ & 515 & 139 & 249 & 127 & $0.51(0.48-0.54)$ & $0.49(0.46-0.52)$ \\
\hline \multicolumn{8}{|c|}{ Subjects with coronary angiography } \\
\hline No MI & $61.4 \pm 10.2$ & 1003 & 282 & 494 & 227 & $0.53(0.51-0.55)$ & $0.47(0.45-0.50)$ \\
\hline$\geqslant 1 \mathrm{MI}$ & $62.2 \pm 9.6$ & 845 & 206 & 398 & 241 & $0.48(0.46-0.50$ & $0.52(0.50-0.55)$ \\
\hline
\end{tabular}

Coronary angiography was performed by the Judkins method. Vessels were defined as diseased if at least 50\% stenosis were demonstrated. Acute myocardial infarction were diagnosed according to criteria by the World Health Organisation. No vessel disease: Persons without any detectable stenosis of coronary arteries or patients with less than $50 \%$ stenosis of coronary arteries.

stricted to individuals with a high coronary risk profile (Fig. 2). This observation applies to individuals (i) with high apoB levels ( $\geqslant 1.2 \mathrm{~g} / \mathrm{l}$; mean value), (ii) with high Lp(a) levels ( $\geqslant 26 \mathrm{mg} / \mathrm{l}$, mean value), (iii) with high glucose levels ( $>108 \mathrm{mg} / \mathrm{dl}$, mean value), (ii) with a combination of high apoB and Lp(a) levels (mean values, respectively), and (iv) with a combination of high apoB, Lp(a) and glucose plasma levels (mean values, respectively) (Fig. 2).

\subsubsection{Risk of myocardial infarction}

In the total sample and also in low and high risk populations, the stromelysin $5 \mathrm{~A} / 6 \mathrm{~A}$ promoter polymorphism was not associated with the risk of MI (Table 2). This observation was made when healthy individuals and also angiographically controlled subjects without MI were compared with MI patients (Table 2). In addition, no significant differences for the $5 \mathrm{~A}$ and $6 \mathrm{~A}$ allele frequencies were calculated when angiographically controlled individuals without MI were compared with MI patients in subgroups of participants with no, single, double and triple vessel disease (Table 3).

\section{Discussion}

In their Editorial [23], Libby and Lee stressed that "... far from being mere structural cement that surrounds cells, the extracellular matrix is a dynamic, interactive milieu that sends signals that influence such critical cell functions as reproduction, life, and death". The extracellular matrix breakdown is strictly con- trolled by matrix metalloproteinases (MMPs) and their inhibitors TIMPs (tissue inhibitors of MMPs). MMPs and TIMPs are under tight transcriptional control; in addition, MMPs are first synthesized as inactive zymogene precursors [24,25]. Thus, the actual activity of MMPs depends on the rate of synthesis, activation, and the balance between active enzyme and inhibitors [23].

The presence of stromelysin-1, a MMP with a broad substrate specificity [26], was originally demonstrated by in situ mRNA hybridisation [11]. A common polymorphism in the stromelysin promoter, located about $600 \mathrm{bp}$ upstream from the start of transcription and defined by the presence of either six (6A) or five (5A) adenosines, has been identified by Ye et al. [12]. The about two-fold higher transcription activity of the $5 \mathrm{~A}$ allelic stromelysin-1 promoter [13] is apparently caused by a preferential binding of a transcription repressor to the $6 \mathrm{~A}$ allele $[14,15]$. It can be concluded that $6 \mathrm{~A}$ allele carriers might have lower stromelysin-1 levels and thus a lower proteolytic activity which favours deposition of extracellular matrix in the atherosclerotic lesion, development of an atherosclerotic plaque and consequently a more rapid progression of coronary stenosis. Not only three earlier studies $[13,16,17]$ but also the present investigation support this hypothesis since we were able to detect an association of the $6 \mathrm{~A}$ allele with the severity of coronary heart disease defined by the Gensini score [19]. Obviously, the 6A allele influence the degree of CHD in a dose-dependent manner: 6A homozygotes had highest, 6A heterozygotes intermediate and 5A5A genotypes lowest CHD scores in the total study sample (Fig. 1) and in subgroups of 


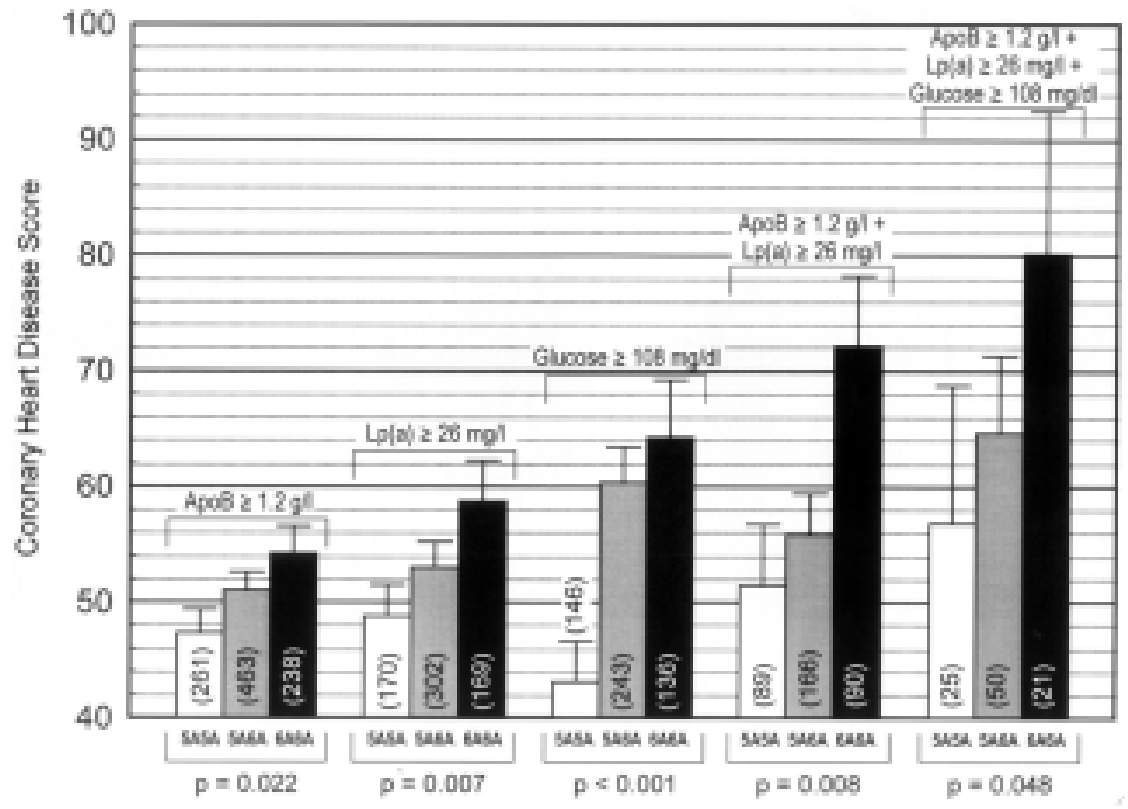

Fig. 2. Comparisons of mean coronary heart disease scores between the stromelysin-1 promoter 5A/6A genotypes in subgroups of patients with coronary high-risk profiles. The mean CHD scores were compared by multiple regression analysis after adjustment for coronary risk factors between patients who underwent coronary angiography for diagnostic purposes. Values are means \pm SEM. Numbers of individuals are given in parentheses. The mean values of coronary risk factors were used as cut points for definition of high risk populations. Coronary angiography was performed by the Judkins method. The CHD score was estimated according to Gensini [19]. A two-tailed probability value of $<0.05$ was considered to indicate statistical significance.

Table 3

Comparison of stromelysin-1 5A and 6A genotypes between individuals without MI and MI patients

\begin{tabular}{|c|c|c|c|c|c|c|c|c|c|c|c|c|}
\hline & \multirow[t]{2}{*}{$\mathrm{n}$} & \multicolumn{3}{|c|}{$-\mathrm{MI}$} & \multicolumn{3}{|c|}{$+\mathrm{MI}$} & \multicolumn{4}{|c|}{ 5A/6A Alleles $( \pm \mathrm{MI})$} & \multirow[t]{2}{*}{$2 p$} \\
\hline & & $\begin{array}{c}\mathrm{n} \\
5 \mathrm{~A} 5 \mathrm{~A}\end{array}$ & $\begin{array}{c}\mathrm{n} \\
5 \mathrm{~A} 6 \mathrm{~A}\end{array}$ & $\begin{array}{c}\mathrm{n} \\
6 \mathrm{~A} 6 \mathrm{~A}\end{array}$ & $\begin{array}{c}\mathrm{n} \\
5 \mathrm{~A} 5 \mathrm{~A}\end{array}$ & $\begin{array}{c}\mathrm{n} \\
5 \mathrm{~A} 6 \mathrm{~A}\end{array}$ & $\begin{array}{c}\mathrm{n} \\
6 \mathrm{~A} 6 \mathrm{~A}\end{array}$ & $5 \mathrm{~A}(-\mathrm{MI})$ & $5 \mathrm{~A}(+\mathrm{MI})$ & $6 \mathrm{~A}(-\mathrm{MI})$ & $6 \mathrm{~A}(+\mathrm{MI})$ & \\
\hline NVD & 432 & 100 & 177 & 86 & $\overline{22}$ & 35 & 12 & ) & F & 0. & 1) & 0.260 \\
\hline & 3 & 62 & 87 & & 39 & 73 & 5 & & & & & 0.121 \\
\hline DVD & 396 & 56 & 9 & 45 & 45 & 9. & 61 & ) & 5( & 0. & 0.54 & 0.095 \\
\hline TVD & 661 & 64 & 136 & 53 & 100 & 329 & 168 & $0.52(0.48-0.56)$ & $0.48(0.45-0.52)$ & $0.48(0.44-0.52)$ & $0.52(0.48-0.55)$ & 0.274 \\
\hline
\end{tabular}

Stromelysin-1 5A/6A genotypes were compared between angiographically controlled individuals without MI and MI patients in subgroups of participants with no, single, double and triple vessel disease. Coronary angiography was performed by the Judkins method. Vessels were defined as diseased if at least $50 \%$ stenosis were demonstrated. Acute myocardial infarction were diagnosed according to criteria by the World Health Organisation. No vessel disease: Persons without any detectable stenosis of coronary arteries or patients with less than 50\% stenosis of coronary arteries. Abbreviations: NVD, no vessel disease; SVD, single vessel disease; DVD, double vessel disease; TVD, triple vessel disease; 2p, two-tailed p-values for the comparisons between individuals without MI and MI patients by multiple logistic regression.

high risk patients (Fig. 2). Humphries et al. [16] stated that - since measurements of the stromelysin-1 expression level in atherosclerotic plaques are essentially impossible - the stromelysin-1 5A/6A promoter polymorphism may act as a disease marker for coronary heart disease and add information to measurable plasma risk factors for estimating the severity and prognosis of this disease. The potential link of the gene variation to the risk of CAD has not been studied so far. Obviously, both alleles have no impact on the risk of coronary artery disease, since the allele frequencies of $5 \mathrm{~A}$ and
6A were essentially the same in healthy controls and patients with coronary heart disease (see Table 2). This observation applied to the total sample and to subpopulations of individuals with low- and high coronary risk profile.

In contrast and due to the about two-fold higher transcription activity of the $5 \mathrm{~A}$ allelic stromelysin-1 promoter which leads to a higher rate of remodelling and degradation of matrix components, it was hypothesized that $5 \mathrm{~A} / 5 \mathrm{~A}$ homozygotes may have an increased risk of plaque rupture and subsequent myocardial infarc- 
tion [18]. The identification of such functional gene variations would achieve a great advantage in the prevention of MI by distinguishing patients who have genetically increased susceptibility to plaque rupture. Indeed - in a Japanese population - Terashima et al. identified the 5A allele as an independent risk indicator of myocardial infarction [18]. To our knowledge, other studies analysing the relation of the $5 \mathrm{~A} / 6 \mathrm{~A}$ promoter polymorphism to MI have not been studied so far. The results of the present study clearly indicate that the $5 \mathrm{~A}$ allele carriers were not over-represented in the group of MI patients (Table 2). This conclusion could be drawn for the comparison of healthy controls to MI patients and of angiographically controlled subjects without and with MI when univariate (data not shown) or multivariate (Table 2) statistical procedures were applied. Regarding the functional characteristics of the $5 \mathrm{~A}$ allele and the strong association of the established coronary risk factors with MI (Table 1), we speculated that the functional importance of the $5 \mathrm{~A}$ allele or of the $5 \mathrm{~A} 5 \mathrm{~A}$ genotype may only become apparent in subgroups of study participants with similar degree of vessel disease. Therefore, we compared the 5A/6A allele frequencies between angiographically controlled patients without and with MI in subpopulations of individuals with zero, single-, double or triple vessel disease. Also these comparisons did not reveal any significant association of the $5 \mathrm{~A}$ allele with the risk of MI (Table 3 ). In consequence, the results of our study strengthen the hypothesis that - in a German population - the 5A allele and/or the 5A5A genotype has no significant impact on the risk of MI. Further studies are warranted to add information to the important question whether the $5 \mathrm{~A}$ allele or the 5A5A genotype might act as disease marker for an increased susceptibility for MI caused by plaque rupture.

\section{Conclusions}

Although the present results do not confirm an association of the $5 \mathrm{~A}$ allele with the risk of MI, they strengthen the hypothesis of earlier investigations that the $6 \mathrm{~A}$ allele is a disease marker for progression of coronary heart disease. Further studies should evaluate whether 5A6A heterozygotes and 6A6A homozygotes - especially with a coronary high-risk profile might benefit from a more aggressive therapy against CHD progression.

\section{References}

[1] R.A. Hegele, The pathogenesis of atherosclerosis, Clin Chim Acta 246 (1996), 21-38.

[2] R. Ross, The pathogenesis of atherosclerosis: a perspective for the 1990s, Nature 362 (1993), 801-809.

[3] R. Ross, Atherosclerosis - An inflammatory disease, New Engl J Med 340 (1999), 115-126.

[4] P. Libby, From Bench to bedside: molecular basis of acute coronary syndromes, Circulation 91 (1995), 2844-2850.

[5] E. Falk, P.K. Shah and V. Fuster, Coronary plaque disruption, Circulation 92 (1995), 657-671.

[6] P.K. Shah, E. Falk, J.J. Badimon, A.F. Ortiz, A. Mailhac, G.V. Levy, J.T. Fallon, J. Regnstrom and V. Fuster, Human monocyte-derived macrophages induce collagen breakdown in fibrous caps of atherosclerotic plaques, Circulation 92 (1995), 1565-1569.

[7] Z.S. Galis, G.K. Sukhova, M.V. Lark and P. Libby, Increased expression of matrix metalloproteinases and matrix degrading activity in vulnerable regions of human atherosclerotic plaques, J Clin Invest 94 (1994), 2493-2503.

[8] A.C. Newby, K.M. Southgate and M. Davies, Extracellular matrix degrading metalloproteinases in the pathogenesis of arteriosclerosis, Basic Res Cardiol 89 (1994), 159-170.

[9] D.L. Brown, M.S. Hibbs, M. Kearney, C. Loushin and J.M. Isner, Identification of $92-\mathrm{kD}$ gelatinase in human coronary lesions. Association of active enzyme synthesis with unstable angina, Circulation 91 (1995), 2125-2131.

[10] S.T. Nikkari, K.D. O'Brien, M. Ferguson, T. Hatsukami, H.G. Welgus, C.E. Alpers and A.W. Clowes, Interstitial collagenase (MMP-1) expression in human carotid atherosclerosis, Circulation 92 (1995), 1393-1398.

[11] A.M. Henney, P.R. Wakeley, M.J. Davies, K. Foster, R. Hembry, G. Murphy and S. Humphries, Localisation of stromelysin gene expression in atherosclerotic plaques by in situ hybridisation, Proc Natl Acad Sci USA 88 (1991), 8154-8158.

[12] S. Ye, G.F. Watts, S. Mandalia, S.E. Humphries and A.M. Henney, Preliminary report: genetic variation in the human stromelysin promoter is associated with progression of coron ary atherosclerosis, Br Heart $\mathbf{J} 73$ (1995), 209-215.

[13] S. Ye, P. Eriksson, A. Hamsten, M. Kurkinen, S.E. Humphries and A.M. Henney, Progression of coronary atherosclerosis is associated with a common genetic variant of the human stromelysin-1 promoter which results in reduced gene expression, J Biol Chem 271 (1996), 13055-13060.

[14] S. Ye, C. Whatling, H. Watkins and A. Henney, Human stromelysin gene promoter activity is modulated by transcription factor ZBP-89, FEBS Lett 450 (1999), 268-272.

[15] R.C. Borghaei, C. Sullivan and E. Mochan, Identification of a cytokine-induced repressor of interleukin-1 stimulated expression of stromelysin-1 (MMP-3), J Biol Chem 274 (1999), 2126-2131.

[16] S.E. Humphries, L.A. Luong, P.J. Talmud, M.H. Frick, Y.A. Kesäniemi, A. Pasternack, M.R. Taskinen and M. Syvänne, The 5A/6A polymorphism in the promoter of the stromelysin1 (MMP-3) gene predicts progression of angiographically determined coronary artery disease in men in the LOCAT gemfibrozil study, Atherosclerosis 139 (1998), 49-56.

[17] M.P. DeMaat, J.W. Jukema, S. Ye, A.H. Zwinderman, P.H. Moghaddam, M. Beekman, J.J. Kastelein, A.J. van Boven, A.V. Bruschke, S.E. Humphries, C. Kluft and A.M. Henney, Effect of the stromelysin-1 promoter on efficiacy of pravastatin in coronary atherosclerosis and restenosis, Am J Cardiol $\mathbf{8 3}$ (1999), 852-856. 
[18] M. Terashima, A. Hozuka, K. Kanazawa, N. Inoue, S. Yamada, I. Keiichi, Y. Matsuda, E. Takai, C. Iwai, H. Kurogane, Y. Yoshida and M. Yokoyama, Stromelysin promoter 5A/6A polymorphism is associated with acute myocardial infarction, Circulation 99 (1999), 2717-2719.

[19] G.G. Gensini, A more meaningful scoring system for determining the severity of coronary heart disease, Am J Cardiol 51 (1983), 606.

[20] D.A. Rose, The diagnosis of ischaemic heart pain and intermittent claudication in field surveys, Bulletin of the World Health Organisation 27 (1962), 645-648.

[21] A. Gardemann, O. Schwartz, W. Haberbosch, N. Katz, T. Weiß, H. Tillmanns, F.W. Hehrlein, W. Waas and A. Eberbach, Positive association of the $B$ fibrinogen $\mathrm{H} 1 / \mathrm{H} 2$ gene variation to basal fibrinogen levels and to the increase in fibrinogen concentration during acute phase reaction but not to coro- nary artery disease and myocardial infarction, Thrombosis and Haemostasis 77 (1997), 1120-1126.

[22] L. Dunleavey, S. Beyzade and S. Ye, Rapid genotype analysis of the stromelysin gene 5A/6A polymorphism, Atherosclerosis 151 (2000), 587-589.

[23] P. Libby and R.T. Lee, Matrix matters, Circulation 102 (2000), 1874-1876.

[24] H. Nagase and J.F. Woessner, Matrix metalloproteinases, $J$ Biol Chem 274 (1999), 21491-21494.

[25] S.J. George, Tissue inhibitors of metalloproteinases and metalloproteinases in atherosclerosis, Current Opinion in Lipidology 9 (1998), 413-423.

[26] G. Murphy and J.J. Reynolds, Extracellular matrix degradation, in: Connective Tissue and Its Heritable Disorders, WileyLiss, New York, 1993, pp. 8154-8169. 


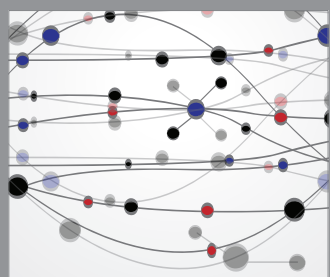

The Scientific World Journal
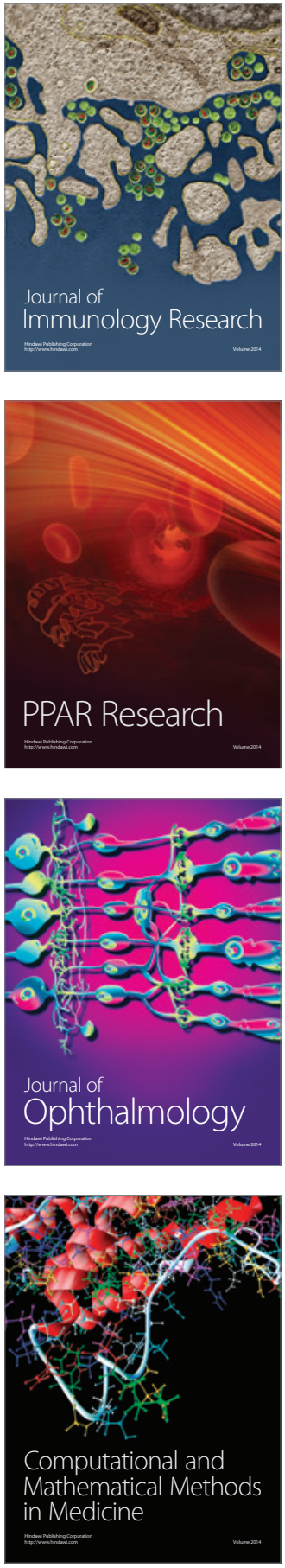

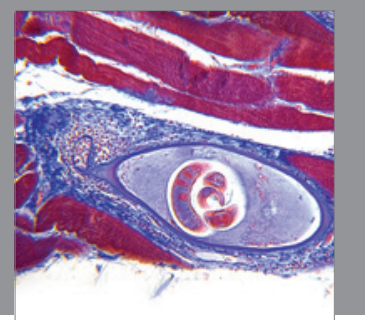

Gastroenterology

Research and Practice
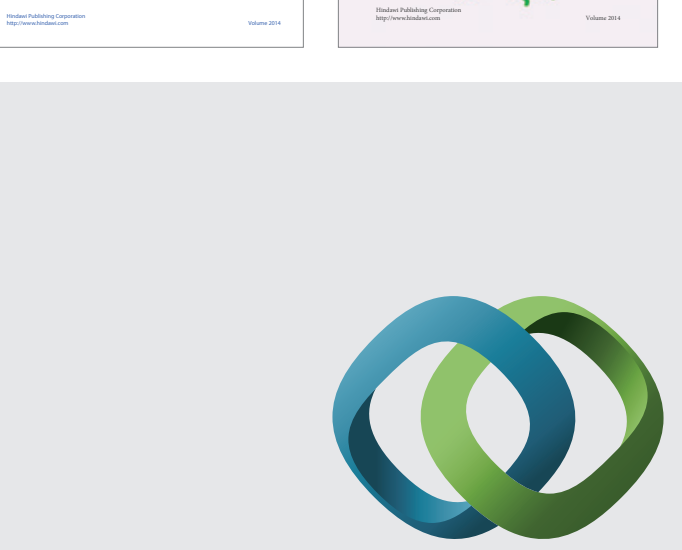

\section{Hindawi}

Submit your manuscripts at

http://www.hindawi.com
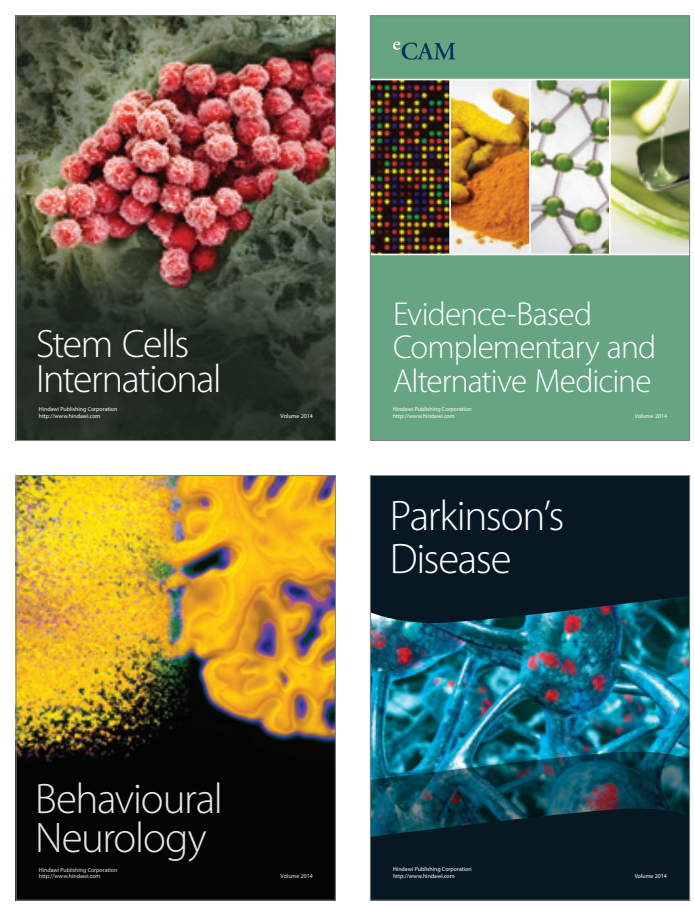

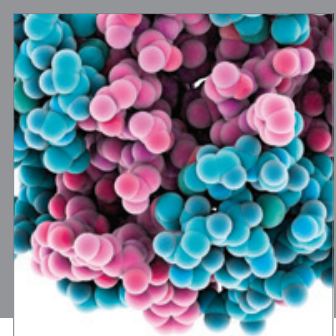

Journal of
Diabetes Research

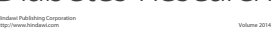

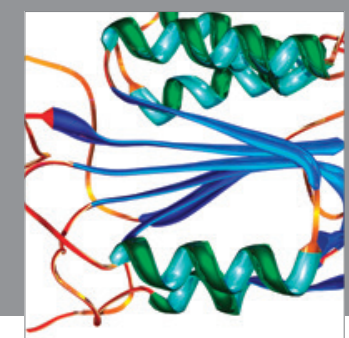

Disease Markers
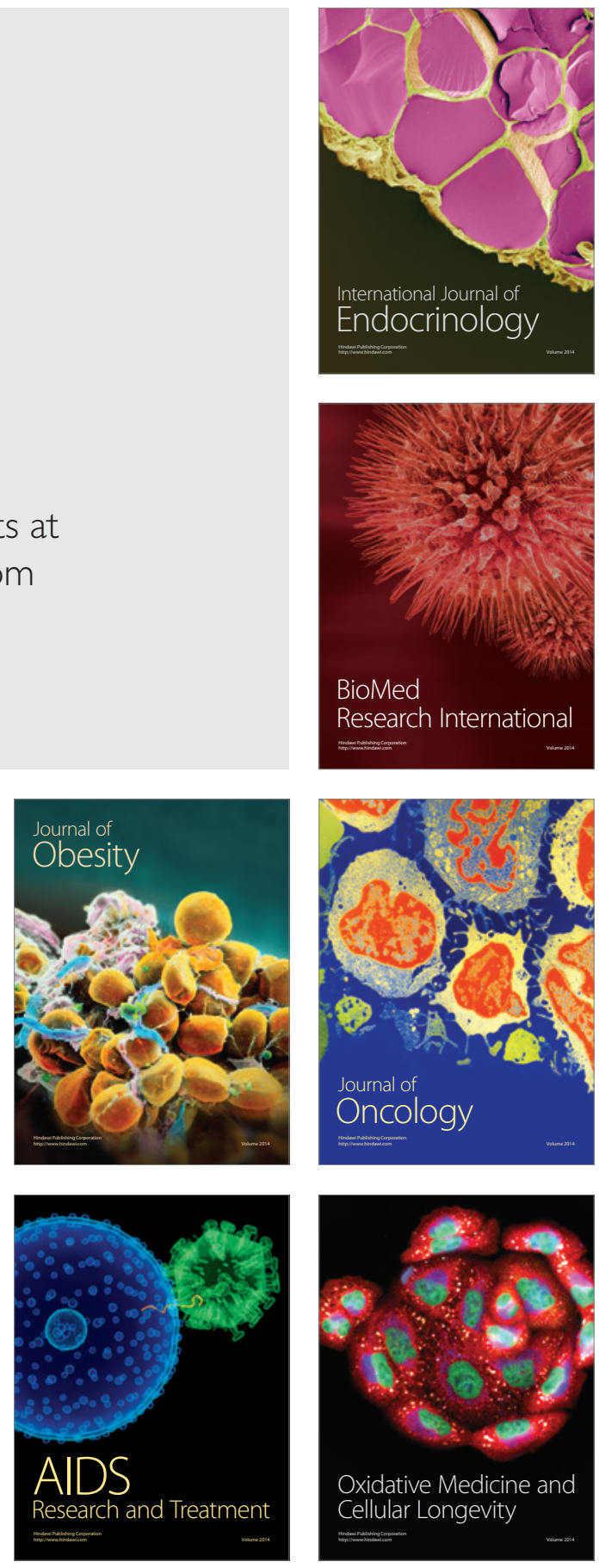